\title{
Cuellos de botella que impiden la reactivación del sector turismo en la región Apurímac, Perú
}

\author{
Bottle Necks that Prevent the Reactivation of the Tourism Sector \\ in the Apurímac Region, Peru
}

\section{FELIPE RAFAEL VALLE DIAZ ${ }^{1}$}

https://orcid.org/0000-0003-0855-9688

\begin{abstract}
RESUMEN: El objetivo general de la investigación fue: Diagnosticar el cuello de botella primordial y complementarios que impiden la reactivación del sector turismo en la región Apurímac, Perú 2020. La investigación está ubicada en el enfoque cualitativo, de tipo básica, el diseño es no experimental, ligado al diseño estudio de caso-descriptivo. El problema primordial es el saneamiento físico-legal de los recursos turísticos, de lo contrario no se podrá obtener dominio, sin ello no se puede inyectar inversión en el entorno local para recuperar, mejorar las instalaciones del recurso turístico. Los complementarios son: la accesibilidad y vulneración del recurso turístico; las limitaciones de jerarquización de los operadores turísticos por no alcanzar requisitos mínimos; la superposición de algunas competencias y funciones de instituciones pertinentes, que hacen duplicar acciones, sumado a la imposición de procesos que frenan el desarrollo del recurso turístico; el direccionamiento e inclinación de crecimiento, expansión de circuitos turísticos en una ciudad de la región Apurímac; y la poca sensibilidad, cooperación para fortalecer el gremio de operadores turísticos.
\end{abstract}

Palabras clave: reactivación; turismo; saneamiento físico-legal.

\begin{abstract}
The general objective of the research was: To diagnose the bottlenecks that prevent the reactivation of the tourism sector in the Apurímac region, Peru 2020. The research is based on a qualitative, basic approach, the design is non-experimental, linked to the study design of case-descriptive. The main bottleneck is the physical-legal sanitation of the tourist resources, otherwise it will not be possible to obtain dominance, without it, investment cannot be injected into the local environment to recover and improve the facilities of the tourist resource. The complementary ones are: accessibility and violation of the tourist resource; the hierarchical limitations of tour operators due to not meeting minimum requirements; the superposition of some competencies and functions of pertinent institutions, which duplicate actions, added to the imposition of processes that slow down the development of the tourist resource; the direction and inclination of growth, expansion of tourist circuits in a city of the Apurímac region; and the low sensitivity, cooperation to strengthen the union of tour operators.
\end{abstract}


Keywords: reactivation; tourism; physical-legal sanitation.

\section{INTRODUCCIÓN}

Según la Comisión Económica para América Latina y el Caribe (Cepal), Latinoamérica tendrá efectos económicos y sociales considerables. Asimismo, la Cepal (2020) precisó que la expansión de la COVID-19, se combatió bajo tres estrategias: autoaislamiento, la cuarentena y el distanciamiento social. Estas decisiones sirvieron para aplanar la curva de contagios, pero sobre todo para dar tiempo a que la logística de los sistemas de salud, se implementen para enfrentar los contagios (p. 2) Está crisis nació cuando existía plena confianza en el proceso de globalización, sin embargo, ya existía un desempeño de la economía mundial que era débil, porque la tasa media de desempeño de crecimiento mundial entre el 2011-2019 fue $2.8 \%$, inferior a la primera década que alcanzó el 3.4\%. A inicios de la expansión de la COVID-19, la tasa de crecimiento anual cayó a 2.5\%. (p. 2)

Del mismo modo, ya no existen estimaciones optimistas cuya baja de la tasa de crecimiento anual sería en 1\%. Según la Cepal (2020) es todo lo contrario, las tasas bordean el intervalo entre el $2.5 \%$ al $4.0 \%$, cuyos resultados se apoyan en los choques de oferta, sostenidos en las medidas de salud pública para contener la COVID-19, lo que degrada la actividad económica por el cierre de fábricas, cese de operaciones de diversos servicios, cancelación de actividades y eventos, paralizaciones y restricciones en los viajes, entre otros, además de ocasionar freno en la cadena de suministros (p.3) Se suma a ello, la reducción del consumo de bienes y servicios, caso del turismo y entretenimiento, dependerá su recuperación de la respuesta de individuos y la reacción respecto al hábito de autoaislamiento y distanciamiento social, por el miedo a los rezagos de contagiados (p.3). Tal es así, que los trabajos se virtualizarían, marcando un giro en las relaciones económicas y sociales, se profundiza el teletrabajo (p. 4) Un impacto para América Latina, es la menor demanda de servicios de turismo, se concretan por las prohibiciones y restricciones de viaje, la contracción podría superar un $25 \%$ (p. 5).

Tocando la situación en Perú, el Ministerio de Comercio Exterior y Turismo Peruano (Mincetur), reportó a marzo 2020 las estadísticas (Turismo-Artesanía, 2020) según Migraciones Perú, en el mes de marzo la llegada de turistas internacionales alcanzó a 114,437 personas, una variación del -69.5\% en comparación al mismo periodo del año 2019; para abril la caída superó el 80\% (p. 1). Además, al primer trimestre del año 2020 la llegada de turistas por el aeropuerto Jorge Chávez se contrajo 23\%, el Complejo Santa Rosa en Tacna cae 26\% y el Complejo en Tumbes cayó 38\% (p. 3).

Es importante, destacar que el gobierno de turno estableció una serie de medidas para apoyar al sector empresas, por el motivo del estado de emergencia y la emergencia sanitaria, Promperú (2020) dentro de lo tributario se prorrogó la Declaración Jurada Anual del Impuesto a la Renta del ejercicio 2019, para tercera categoría con ingresos netos hasta s/. 9,660,000 soles o 2300 Unidades Impositivas Tributarias (UIT) para la tercera categoría con ingresos netos hasta s/. 21,000,000 soles o 5000 UIT. También se prorrogó la Declaración y pago del PDT Planilla electrónica del periodo febrero 2020 para tercera categoría con ingresos hasta s/. 9,660,00 soles y s/. 21,000,000 soles. Para ambos casos también se prorrogó 
la Declaración y Pago de Obligaciones Mensuales de Impuesto General a las Ventas (pp. 4-12), asimismo, en materia financiera Promperú (2020) las empresas formales contenidas en el sector turismo podrían acceder al Programa Fondo de Apoyo Empresarial a la MYPE, conocido como FAE-MYPE, cuyo monto a distribuir fue s/ 300 millones de soles, sostiene nuevos créditos y la reestructura o refinanciamiento de deudas (p. 18) El segundo Programa REACTIVA Perú, cuyo monto a distribuir fue s/ 30 Mil Millones, sostiene nuevos créditos para capital de trabajo (pp. 23-25)

A pesar de la política económica de reactivación, sostenida para apoyar e impulsar el sector empresarial, específicamente para el turismo, estos programas no pudieron llegar en una primera oportunidad, a los negocios contenidos en el sector turismo; por los requisitos que deben alcanzar como el monto de UIT e inclusive por una considerable cantidad de informales, operadores no jerarquizados, con problemas de licencia de funcionamiento, entre otros. A finales de octubre del 2020, después de 240 días de cuarentena; el Gobierno Regional de Apurímac, inicia un programa de Apoyo Financiero para este sector, que, a noviembre del 2020, sigue en la etapa de difusión, capacitación.

Sin embargo, en Perú, a nivel de la sierra peruana, basado en una entrevista a ex director Regional de Comercio Exterior y Turismo de Apurímac ${ }^{2}$, existen muy pocos productos turísticos locales, que tengan una planta de soporte completa, que armonice con la cultura, identidad, tecnología, nos referimos como referencia a las ciudades de Lima y Cusco, porque el resto de recursos y atractivos turísticos, presentan limitaciones, barreras, que generalmente están relacionados a normativa legal, deficiencia en gestión local y sub nacional, que no coadyuva a mejorar las condiciones de despegue, estas limitaciones para el sector turismo, son conocidas en el lenguaje natural como "cuello de botella", que significa; "obstruir", cuyo concepto es; aquel proceso o requisito, que todo recurso turístico necesita para su categorización y jerarquización, cuya evidencia, impide concretar el desarrollo de un recurso turístico. Para este grupo de recursos y atractivos no llega o no puede acceder a los Programas FAE y REACTIVA.

Valle (2020) indica que en la región Apurímac, aquellas provincias que cuentan con un potencial de recursos turísticos únicos, incomparables, se han preservado por causa de las condiciones geográficas, accesibilidad restringida, cuidado de la comunidad campesina, por ello existen las deficiencias en la actualización de las fichas de inventario de los recursos turísticos, sumado a que no existe saneamiento físico-legal (p. 320). El mismo autor advierte la existencia de barreras para mejorar o impulsar un recurso turístico, tales como: debe tener ficha de inventario a la par el saneamiento físico-legal, contar con estadísticas de frecuencia de visitas al recurso turístico, la predisposición del alcalde municipal para concretar gestiones de intervención para recuperación y puesta en valor, entre otros (p. 320), sumado que se identifica una considerable cantidad de recursos turísticos con potencial, con serias limitaciones para impulsarlos (pp. 308-315). A la fecha el inventario de recursos turísticos el instrumento de recojo de datos; la ficha de inventario, el formato de llenado y requisitos fueron modificados, no ha extremos, integrando una sección sobre el saneamiento físico-legal, soporte fotográfico y documentario, precisión sobre los servicios de planta de soporte próximos y alejados al recurso turístico (Mincetur, 2018).

2. Mag. Lisbeth Salas Ccente, ex Director Regional de Comercio Exterior y Turismo, periodo de gestión 2018-2020. Ex director Sub Regional de Comercio Exterior y Turismo sede Andahuaylas, gestión 2017-2018. 
Dado el contexto donde se desarrolla la investigación, la delimitación centrada en tratar las obstrucciones al despegue y crecimiento de recursos turísticos del sector turismo, la posibilidad de su aprovechamiento, se formuló la siguiente pregunta general que direcciona la investigación: ¿Cuál es el cuello de botella primordial y complementarios que impiden la reactivación del sector turismo en la región Apurímac, Perú 2020? Por consiguiente, el objetivo general que permitirá estructurar, precisar el diseño de investigación, es el siguiente: Diagnosticar el cuello de botella primordial y complementarios que impiden la reactivación del sector turismo en la región Apurímac, Perú 2020. El propósito de la investigación es: precisar los procesos o requisitos, que necesariamente deben tener evidencias, que obstruyen, frenan, la reactivación del sector turismo en la región Apurímac. Asimismo, los resultados permitirán conocer el estado actual del sector turismo y servirá para que los funcionarios responsables de la política sectorial, tomen conciencia, coadyuvando a formular políticas, estrategias, objetivos, metas; viables y sostenibles, para la recuperación próxima del sector turismo en la región Apurímac, mermado por las medidas tomadas para frenar la propagación y fallecimientos de ciudadanos por la COVID-19.

\section{MARCO TEÓRICO}

La Organización Mundial de Turismo (OMT) coincide en apreciaciones con el grupo Biosfhere del Instituto de Turismo Responsable, Biosfhere-OMT (2020) en su propuesta de actuación para la recuperación empresarial del sector turístico, nos indica que se deben centrar en lo que pueden controlar en el negocio, aprovechar las variables a nuestro alcance para volver a empezar mejor y más fuerte (p. 1) La propuesta se centra en cuatro estrategias de sostenibilidad, donde el empresario debe adaptarse, transformar y re-actuar en busca de la viabilidad económica.

Para Biosfhere-OMT (2020) la sostenibilidad económica, significa mantener vivo los canales de comunicación, promoción, elaborar un mapa de planificación estratégica donde se precise la gestión de ingresos y control de costos, tomando en cuenta tres escenarios de recuperación; rápida de 2 a 3 meses; media de 3 a 6 meses y lenta mayor a 6 meses; negociar con clientes que separaron cupos o paquetes para una reprogramación con servicios adicionales, gestionar con proveedores la reducción de algunos costos y reprogramar compromisos de corto plazo en mutuo acuerdo; integrar nuevos socios para recuperar el negocio con mirada de diversificación, relanzamiento y mantener la marca del negocio en base a las alianzas socios nuevos y renegociación con proveedores (pp. 8-12).

La sostenibilidad sociocultural, significa estrechar vínculos, relaciones con el personal apoyados de actividades en línea; realizar reuniones para fortalecer el conocimiento y comunicación con empleados sobre el impacto de la crisis; escuchar las sugerencias, para mejorar estrategias y procedimientos, a los clientes proporcionar información sobre el medio ambiente, los valores e identidad sociocultural del destino además del papel de la empresa con el medio ambiente (pp. 20-24).

Sobre la sostenibilidad ambiental, se trata de desarrollar videos o documentales brindando información sobre el papel alcanzado por la empresa, sus intervenciones y pruebas para los clientes, mostrarles los procedimientos amigables con el medio ambiente que abraza la empresa (pp. 27-29) y la sostenibilidad sanitaria, es reforzar la cultura de prevención al 
contagio a partir de una mayor comunicación interna y externa con clientes y proveedores, establecer planes de higiene de ambientes-fumigaciones-señalización, tener ambientes para aislamiento temporal o preventivo, realizar simulacros (pp. 30-34)

Prosiguiendo, el Mincetur, tomando como dirección y apoyo a la política del sector, las recomendaciones de la OMT, propuso unas consideraciones para programas de asistencia técnica para la recuperación del Turismo ante la crisis de la COVID-19, para ello, es difícil recuperar en el corto plazo el sector turismo (OMT, 2020). Hasta la fecha, la pandemia causó la caída del 22\% de las llegadas de turistas internacionales al primer trimestre 2020, la crisis alcanzó una caída del 60\% anual en comparación de las cifras del 2019 (p. 2), este programa se estructura en tres pilares fundamentales: económico, marketing y promoción, fortalecimiento institucional y mejora de la resiliencia.

Por lo tanto, el resumen de las estrategias sugeridas por la OMT (2020) es la siguiente: La primera se basa en estudios para la recuperación económica, el apoyo a las empresas a través de mecanismos como impuestos, tasas, la reorientación de la cadena de valor basado en la identificación de oportunidades financieras para fortalecer los eslabones de la cadena de valor, todos contribuyen asegurar la sostenibilidad, estas acciones están enmarcadas dentro de los objetivos de Desarrollo Sostenible respecto al fin de la pobreza, trabajo decente y crecimiento económico, la reducción de las desigualdades (pp. 5-9). La segunda es la revisión de estrategias de marketing basado en la identificación de mercados que puedan recuperarse rápidamente $\mathrm{y}$ permitan diversificar los productos a partir de promociones planificadas y minimizar la dependencia de una única actividad o mercado, además de incidir en la expansión de actividades innovadoras de marketing digital, igual al anterior estas acciones están enmarcadas dentro de los objetivos de Desarrollo Sostenible respecto al fin de la pobreza, trabajo decente y crecimiento económico, la reducción de las desigualdades, ciudades y comunidades sostenibles, industria-innovación e infraestructura (pp.10-15). La tercera estrategia, es el trabajo conjunto entre Gobierno y empresas para mejorar los servicios que presta la planta de soporte turística en materia de salud, seguridad en el trabajo, promover gobernanza turística, las alianzas público-privadas, mesas de concertación de esfuerzos conjuntos, estas acciones también se encuentran enmarcadas dentro de los objetivos de Desarrollo Sostenible, respecto al trabajo decente y crecimiento económico, educación de calidad, Igualdad de género, producción y consumo responsables, ciudades y comunidades sostenibles y la alianza para lograr los objetivos (pp. 17-21).

Además, el MINCETUR a través de Promperú (2020) consideró pertinente capacitar mediante webinar a gremios empresariales, para afrontar la crisis y poscrisis; centró sus recomendaciones en soluciones innovadoras que satisfagan nuevas necesidades de consumo (p. 1) Para ello es importante proseguir con el trabajo de posicionamiento de marca, debe permanecer en la mente del cliente, ello se logra a través de medios digitales como redes sociales o páginas web. Urgente hacer un diagnóstico que permita conocer la situación actual del negocio, para re direccionar a la diversificación de servicios, los nuevos mercados deben sostenerse en la diversificación iniciada, fortaleciendo las propuestas de agregación de servicios en los paquetes o productos turísticos (p. 3). 


\section{METODOLOGÍA}

Esta investigación es de enfoque cualitativo, por las características de la variable de estudio (Hernández et al., 2014), es una investigación de tipo básica, que permite aportar conocimiento actual de la variable de estudio (Carrasco, 2005). El diseño es no experimental, ligado al diseño estudio de caso-descriptivo, porque analiza a partir de la descripción los procesos o requisitos que impiden despegar al sector turismo (Ortiz \& García, 2008). Las técnicas de investigación fueron: La entrevista y análisis de documentos (Fidias, 2012). Los instrumentos de recojo de datos fueron: ficha de entrevista y lista de cotejo documentos. Las unidades de análisis fueron los recursos turísticos, bibliografía existente y la normativa vigente.

\section{RESULTADOS}

Los resultados los obtenemos a partir de la experiencia, observación in situ, entrevistas a funcionarios, análisis de documentos de gestión, y Leyes 24656, 24657, Resolución Ministerial N 493-2018 de Reglamento de Intervención Arqueológica del Ministerio de Cultura-Perú, Plan Estratégico Regional de Turismo Apurímac 2018-2025, la Guía para actualización e inventario de recursos turísticos del Ministerio de Comercio Exterior y Turismo-Perú.

a) El primer cuello de botella, es la propiedad del recurso turístico

La propiedad de un bien es determinante para poder iniciar acciones, disponer del bien; sin embargo, existe un problema, la mayoría de recursos turísticos, no cuentan con saneamiento físico-legal, por tal motivo la propiedad no se encuentra definida, no hay un dominio pleno por un propietario, por lo cual todos los recursos turísticos quedan en resguardo de la población donde se ubica el recurso turístico. Por lo general, se encuentran en territorios comunales campesinos, los cuales están inscritos en Registros Públicos.

Además, existen otros problemas en el proceso de saneamiento físico-legal, como: cuando se realiza la delimitación y deslinde de una porción de terreno que contiene el recurso turístico, acontecen varias situaciones que son generadas en el proceso como:

1. Respecto a la delimitación y deslinde de linderos, el levantamiento perimétrico de la extensión de terreno que contiene el recurso turístico, no contó con acta de aprobación por la asamblea general de la comunidad campesina, en muchas ocasiones solo contó con aprobación a nivel de la directiva de la comunidad campesina, lo cual no es suficiente, porque obstruye todo el proceso de saneamiento.

2. Respecto que, la asamblea general de la comunidad campesina, aprobó entregar el terreno que contiene el recurso turístico, sin haber tomado conocimiento del área y perímetro, aprobando a ciegas e inclusive sin haber recompensado o devuelto terrenos a colindante o posesionario; esto impide todo el proceso de saneamiento. 
3. Cuando una institución pública con competencia y funciones respecto a lo que contiene el recurso turístico, obtiene el permiso de la asamblea comunal para realizar la delimitación y deslinde además del levantamiento perimétrico, pero en asamblea comunal aprueban solo el terreno o terrenos que ocupa el recurso turístico; sin embargo, la institución bajo sus criterios y con la aprobación de asamblea, ejecuta la delimitación y deslinde, levantamiento perimétrico, abarcando más terrenos de los especificados por la asamblea comunal; esto también genera inestabilidad, conflictos y frena el proceso de saneamiento.

4. Cuando el comunero calificado desde los padres, cuenta con años de posesión, explotación continua, usufructuando en forma pública, cuya frontera agrícola la ha mantenido, en muchas ocasiones instituciones por competencia y función, pretende tomar la porción de terreno para el saneamiento, sin entregar un justiprecio, de tal forma que el comunero reciba una retribución económica por dejar su fuente de manutención; sino se entrega una retribución al comunero que se pretenda tomar su terreno; entonces estos conflictos también obstruye el proceso de saneamiento, ello sucede cuando en las comunidades campesinas, ya no se cuenta con terrenos libres para realizar el canje.

5. Sucede casos en los cuales la asamblea de la comunidad campesina, sin tener pleno conocimiento, haber llevado un control; decide tomar los terrenos donde se ubica y contiene el recurso turístico, despojando a comuneros no calificados; los cuales, tienen derechos de posesión, usufructo por tiempo considerable e inclusive muchos tienen familia en la comunidad; esto sucede, cuando contraen matrimonio una pareja donde cada uno es proveniente de diferente comunidad; al despojarlos, cometen abuso de autoridad, desconocen la posesión, usufructo permitido, sin presentar sanciones los despojados; entonces el terreno entregado a una institución pública aprobado por la asamblea general; trae consecuencias, el despojo genera conflictos y asciende hasta demanda judicial, se frena todo el proceso de saneamiento.

6. La sexta; cuando las instituciones públicas con competencia y funciones, reciben el permiso por parte de la asamblea comunal, cumpliendo con los requisitos previos antes de iniciar el proceso para la delimitación y deslinde, levantamiento perimétrico; lo realizan, sin embargo, cuando se contrasta o replantea el plano y acta de colindancia en campo, sucede que se ubica en otra posición; esto también generó conflictos, pérdida de confianza de la comunidad con la institución, obstruyéndose el proceso de saneamiento. Lo primordial en estos casos, es que se debe reconocer la norma vigente en la que se debe apoyar la delimitación y deslinde, levantamiento perimétrico del terreno que contiene el recurso turístico es la Ley N 24656 Ley General de Comunidades Campesinas y la Ley N² 24657 Ley del Deslinde y Titulación de Territorios Comunales. 


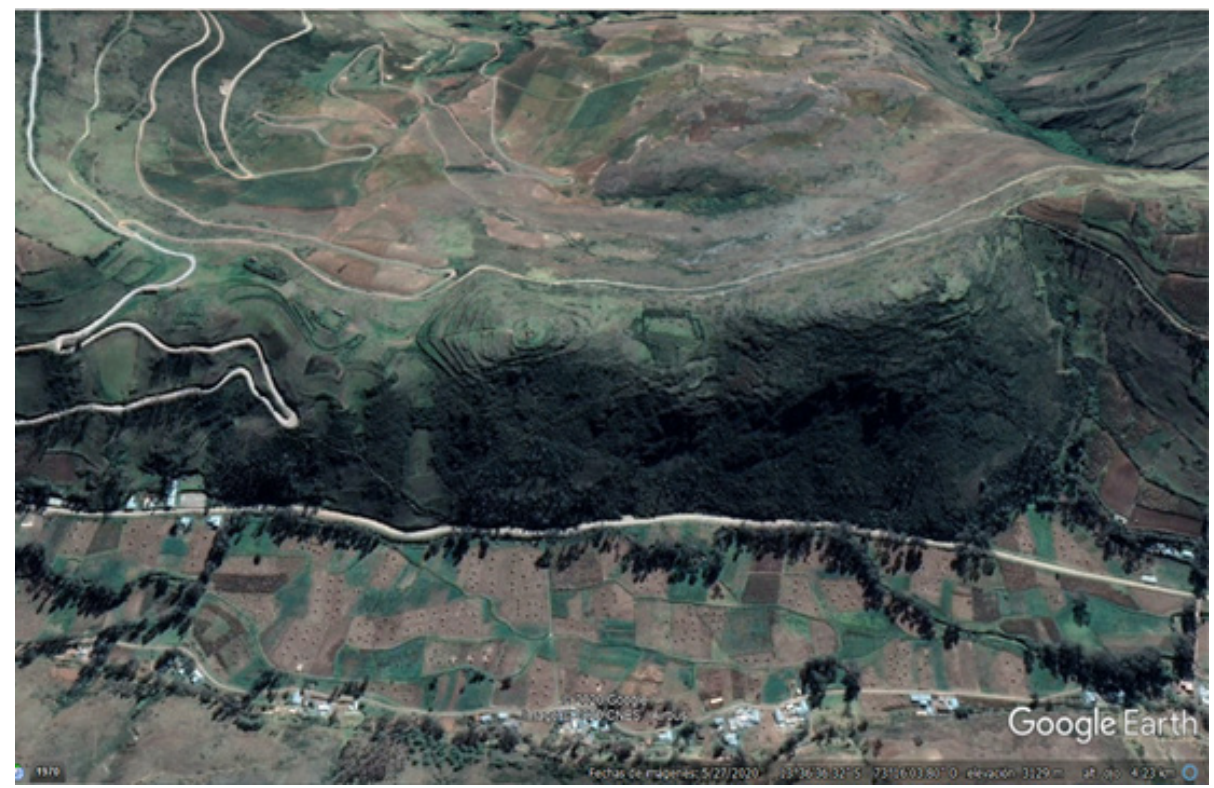

Figura 1. La captura fotográfica muestra el caso tratado sobre el Complejo Arqueológico de Sóndor, ubicado en el distrito de Pacucha, provincia de Andahuaylas, tiene más de 30 años de conducción por Municipio Local, no cuenta con saneamiento físico-legal, presenta administración por Asociación de Operadores, aprobada y entregada por el Municipio Local, a la fecha de la presente investigación, se encuentra en conflicto de funciones con el Ministerio de Cultura. Los trabajos de recuperación y exposición solo están al 10\% de su potencial. Cuenta con dos publicaciones científicas. Está compuesto por sectores ceremonial, vivienda, cementerios, andenerías.

Fuente: Google Earth, 2020. Fecha de imagen 27/05/2020. Coordenadas: Sur 13¹6'27.05", Oeste $73^{\circ} 16^{\prime} 04.93^{*}$, elevación 3214 m, altura ojo 4.23 Km. Recuperado: 28.11.2020.

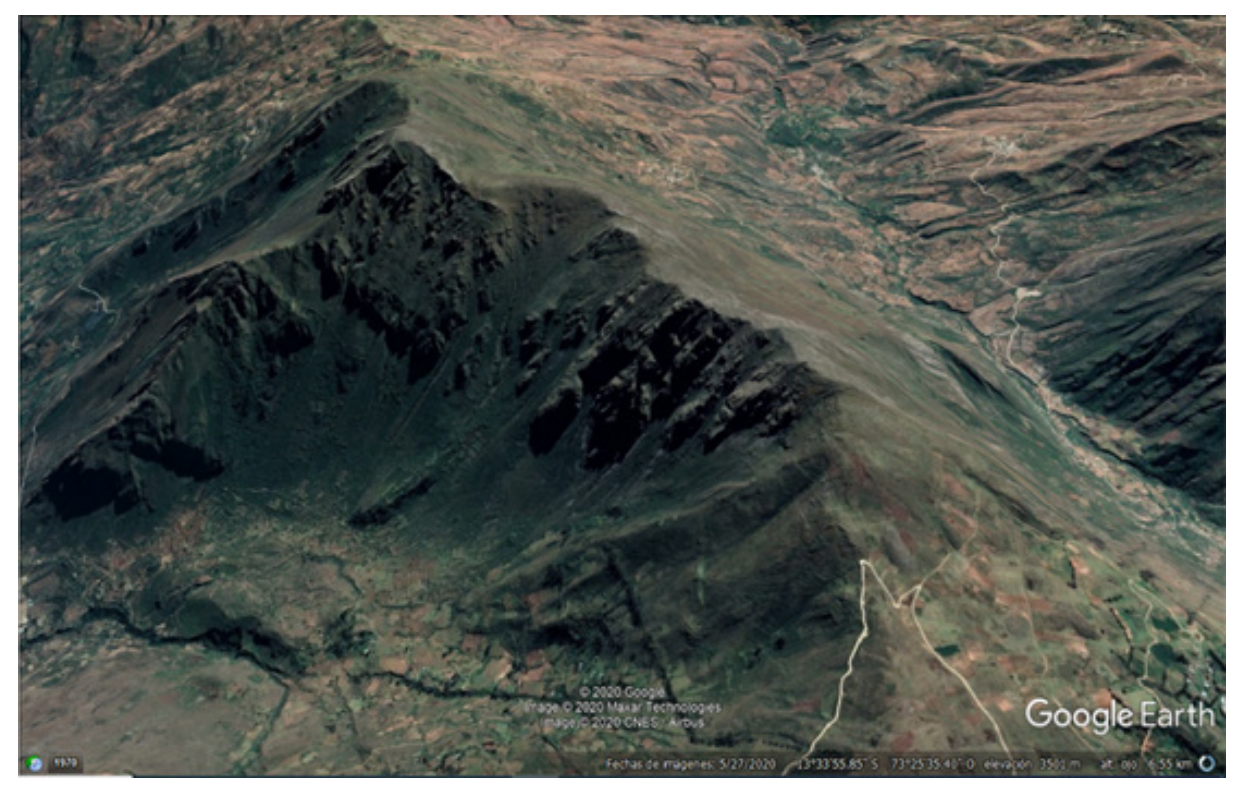

Figura 2. La captura fotográfica muestra el caso sobre el Complejo Arqueológico de Achanchicc, ubicado en el divortium aquarum de los distritos de Pacucha y Talavera, provincia de Andahuaylas, no cuenta con conducción por Municipio Local y saneamiento físico-legal, 
presenta resguardo por la Comunidad Campesina de Uchuhuancaray, a la fecha de la presente investigación no se encuentra en conflicto de funciones con el Ministerio de Cultura. Los trabajos de recuperación y exposición solo están en prospección de su potencial. Existe en el primer sector alta vulneración (huaqueo) por la entrada sur en el Complejo Arqueológico de Achanchicc. Está compuesto por sectores ceremonial, viviendas, almacenes, sistemas de andenería en laderas, canales de abastecimiento de recurso hídrico, cementerio en cuevas (machays).

Fuente: Google Earth, 2020. Fecha de imagen 27/05/2020. Coordenadas: Sur 13³3'57.64", Oeste $73^{\circ} 25^{\prime} 25.88^{\prime \prime}$, elevación 3665 m, altura ojo $6.55 \mathrm{Km}$. Recuperado: 28.11.2020.

Respecto al Reglamento de Intervención Arqueológica, este no define como primera acción de intervención el saneamiento físico-legal, no es determinante para ellos este proceso inicial; intervienen haciendo declaratoria, sin el previo proceso del saneamiento, mantiene una base de sitios arqueológicos por intervenir; sin embargo, no cuentan con normativa específica, para realizar el saneamiento físico-legal, por el contrario la opción que optan es apoyarse en las normativas conexas, debido a que los recursos turísticos del tipo manifestaciones culturales, conocido como sitios arqueológicos, se ubican en los territorios comunales campesinos, inscritos en Registros Públicos, además que estos sitios arqueológicos son protegidos, resguardados por la comunidad campesina sin recibir ninguna retribución económica.

A la fecha es difícil realizar un saneamiento físico-legal completo; en los sitios arqueológicos, porque el Ministerio en competencia y función a través de sus funcionarios, presentan comportamiento de intervención e imposición, desconociendo la posesión, cultura, resguardo, seguridad otorgada por la comunidad campesina, trasgrediendo el territorio, buscando una independización superior afectando el medio de vida de los campesinos.

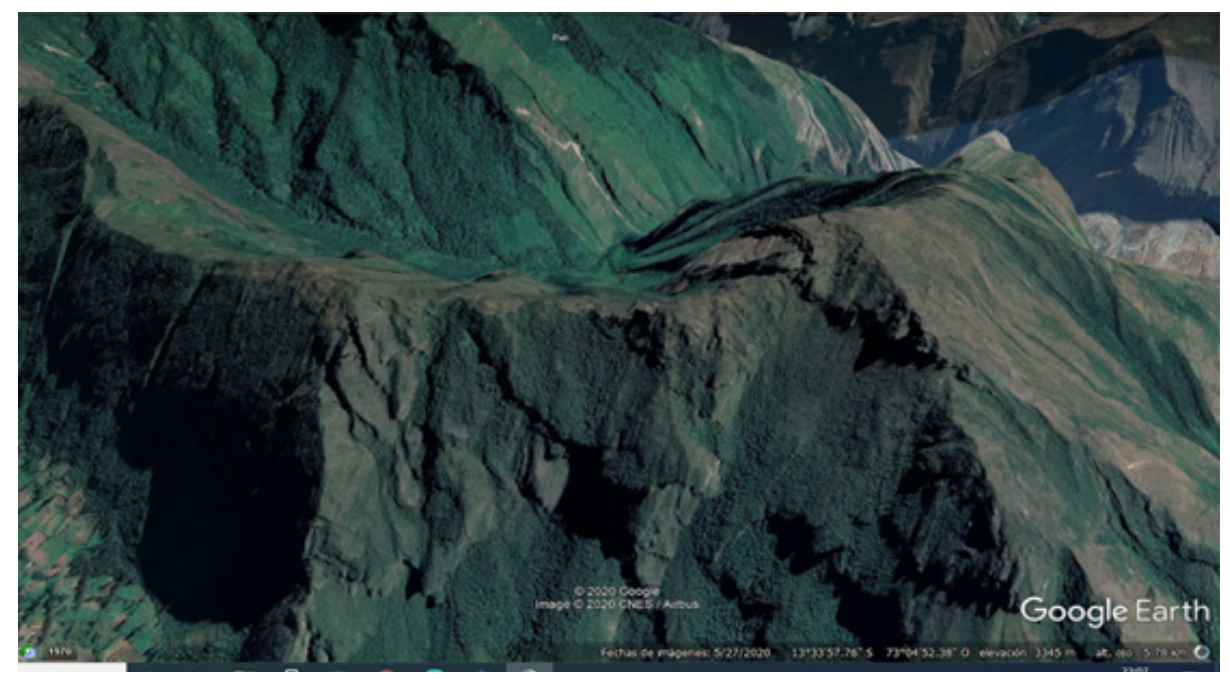

Figura 3. La captura fotográfica muestra el caso sobre el Complejo Arqueológico de Llaqtapata-Qapac ñan-Qoriwayrachina, ubicado en el divortium aquarum de las parcialidades privadas de Pacchani (al sur), Chinchay (al norte), Qoriwayrachina (al este) y Comunidad Campesina Ccerabamba-Andina (al sur) provincia de Andahuaylas, no cuenta con conducción por Municipio Local, el saneamiento físico-legal está en proceso, presenta resguardo 
por la Comunidad Campesina de Ccerabamba-Andina y parcialidad de Pacchani, a la fecha de la presente investigación no se encuentra en conflicto de funciones con el Ministerio de Cultura. Los trabajos de recuperación y exposición solo están en prospección de su potencial. Existe en el primer sector baja vulneración (huaqueo) por la entrada este de la parcialidad de Pacchani. Cuenta con dos publicaciones científicas. Este complejo arqueológico cuenta con camino inca, sitio arqueológico, andenerías, cementerios en cuevas (machays) y centro de fundición.

Fuente: Google Earth, 2020. Fecha de imagen 27/05/2020. Coordenadas: Sur 13³3'41.57"; Oeste $73^{\circ} 04^{\prime} 32.65^{*}$, elevación 3537 m, altura ojo 5.78 Km. Recuperado: 28.11.2020.

Para el caso de inventario de recurso turístico, se establecen coordinaciones entre las instancias de los Ministerios de Comercio Exterior y Turismo con Cultura, el estudio de saneamiento físico-legal incompleto, no permite que se complete la ficha técnica de inventario, porque es un requisito el saneamiento del recurso turístico. En el Plan Estratégico Regional de Turismo Apurímac 2018-2025; no figura como debilidad del sector Turismo, el saneamiento físico-legal, tampoco figura como una estrategia dentro de un objetivo estratégico, si se considera de forma literal dentro de una acción estratégica el saneamiento físico-legal, priorizando aquellos recursos turísticos que estén contenidos dentro del corredor turístico, para realizar intervenciones con proyectos, no cuenta con ningún indicador.

a) El segundo cuello botella es la accesibilidad del recurso turístico, el estado del recurso turístico y la planta de soporte.

Los recursos turísticos en la región Apurímac, que cuentan con jerarquía 3, es decir que presenta infraestructura mínima, registro-control, mantenimiento, una administración formal, son: piedra Saywite, santuario Ampay y el Museo Antropológico en Abancay, el resto de recursos turísticos que existen en la región Apurímac, presentan jerarquía entre 1 y 2 , están en proceso de adecuación y no cuentan con infraestructura mínima. Cabe destacar, que los recursos turísticos ubicados en el rango de 1 y 2 son aquellos que tienen un potencial único, amplio, que por lo general cuentan con poca accesibilidad, referido a trochas carrozables, caminos de herradura, senderos, además cuya planta de soporte próxima cuenta con servicios mínimos relacionados a alimentación, pernoctación del lugar, los alimentos preparados con insumos propios del lugar que da una connotación, peculiaridad por las costumbres de propias; el ambiente de pernoctación es amplio, se comparte porque están las camas separadas contenidas en un solo ambiente general, es un salón amplio convertido en cuarto comunal de pernoctación. Solo los recursos turísticos que están ubicados en el parámetro de jerarquización 2, no se encuentran vulnerados a extremo, es decir tienen un estado de intervención antrópica hasta en un $30 \%$ de afectación al recurso turístico; por lo tanto, se puede recuperar; sin embargo, aquellos que superen el porcentaje deben tener una inspección preliminar para verificar el estado de vulneración y la posibilidad de recuperación.

Respecto al Reglamento de Intervención Arqueológica, es determinante que para ingresar a un recurso turístico tipo manifestación cultural, caso sitio arqueológico o Kapacc Ñan, es necesario el permiso de intervención cuando se pretenda hacer recuperación, cuyas acciones demanden excavación, reconstrucción, instalación, acon- 
dicionamiento de seguridad. Para el caso de inventario de recurso turístico, se establecen coordinaciones entre las instancias de los Ministerios de Comercio Exterior y Turismo con Cultura, el estudio de recuperación si arroja que el recurso turístico está vulnerado en 60\%; es irrecuperable, no procede el inventario del recurso turístico.

En el Plan Estratégico Regional de Turismo Apurímac 2018-2025; figura como debilidad del sector Turismo, el mal estado de las vías de acceso, pero no específica si son las secundarias, interiores o nacional; no es considerado como estrategia, es considerado como una acción estratégica la accesibilidad de las vías de comunicación hacia los recursos o atractivos turísticos, cuenta con un indicador, pero que no es consistente, porque recaba datos sobre el número de recursos turísticos / atractivos priorizados con vías de acceso adecuadas, este indicador es muy general y espera datos de resultados de mediano plazo. El indicador debería recabar datos sobre: número de carreteras internas ampliadas con bicapa que llegan al recurso turístico; otro indicador, número de caminos de herradura o Kapacc Ñan mejorados que llegan al recurso turístico; otro indicador, número de carreteras afirmadas alternas para previsión ampliadas que llegan al recurso turístico.

b) Las competencias y pertinencias funcionales de instituciones inmersas en el sector Turismo.

Un problema mayor a la fecha es sobre la administración y control de sitios arqueológicos, recursos ecoturísticos; donde existe varias instituciones con competencias y función específicas. Las municipalidades provinciales y distritales, que tienen a su cargo; es decir, la administración, control, conservación de un sitio arqueológico o recurso ecoturístico; no pueden realizar mejoras de infraestructura, ampliación de servicios, porque no cuentan con título de propiedad, presentan restricciones en gestión por la inexistencia de planes de desarrollo turístico tanto provincial y distritales; además, no cuentan con planes de desarrollo urbano; por ello, es muy difícil poder reactivar el sector turismo a partir del potencial de recursos turísticos como: sitios arqueológicos, lagos, bosques naturales arbustivos o forestales, caminos incas. Complementa, a todo lo descrito, respecto a la conveniencia de que un recurso turístico sea administrado por el Ministerio en competencia y función o por la función Municipal; este último, por estar ligada y relacionado a la población, existen posiciones de negación; por ejemplo, que el Ministerio de Cultura sea propietario y administre el sitio arqueológico, porque todos los ingresos obtenidos se derivan hacia esta institución, sumado a que no se permite realizar actividades, grabaciones, u otras acciones sin el permiso del Ministerio de Cultura al interior del sitio arqueológico.

Un caso ejemplo es la Municipalidad Distrital de Pacucha, ubicada en la provincia de Andahuaylas, el complejo arqueológico de Sóndor, no es propiedad de nadie, porque no se ha formalizado la independización del territorio que comprende el sitio arqueológico, la posesión la tuvo años atrás el Gobierno Regional de Apurímac, luego fue derivada la posesión hacia la Municipalidad Distrital de Pacucha, hasta la actualidad, quién como posesionario, dio el permiso para la Administración a una asociación de comuneros campesinos, formalizados, que brindan el servicio de registro-control, orientación, alimentación al interior del Complejo Arqueológico de Sóndor. 
Otro ejemplo, es el carnaval Pukllay, años atrás estuvo bajo la dirección y administración del Gobierno Regional de Apurímac, posteriormente pasó a la Municipalidad Provincial de Andahuaylas, aquí en la organización y proceso también interviene la Dirección Sub Regional de Comercio Exterior y Turismo Andahuaylas, duplica funciones como: volantes de publicidad, organización de pre eventos paralelos, realiza filmación y documental, interviene en la bienvenida, recepción, traslado, de participantes extranjeros y nacionales.

Sumado a todo ello, las ciudades principales de la región Apurímac, contenido en el Plan Estratégico Regional de Turismo Apurímac, son Andahuaylas y Abancay, no cuentan con un Plan de Desarrollo Turístico de la provincia, a pesar de la amplia planta de soporte, semi categoriza y jerarquizada, debido a que los locales, ambientes, donde laboran los operadores turísticos cuentan con pocos requisitos para alcanzar un nivel de jerarquización; además está sujeto a la débil planificación y gestión de la infraestructura urbana, porque ambas ciudades no prestan condiciones para un Turismo Social, por lo tanto su categorización internacional es mínima, con brechas a cerrar.

En el Plan Estratégico Regional de Turismo Apurímac 2018-2025; figura como amenaza del sector la pérdida progresiva de la institucionalidad; sin embargo, no consideran que existen competencias similares con otro sector e inclusive las funciones e intervenciones administrativas se superponen, tal es el caso que ambos pretende tener la administración de un recurso turístico o sitio arqueológico, para el MINCETUR, es imperioso que la Administración del recurso quede a cargo de la Municipalidad y se aplique un boleto turístico, cuyo costo tendrá varios componentes, y se podrá atender las necesidades diversas no solo del sitio arqueológico sino además de operadores directos, comunidades vecinas protectoras; para Cultura, es competencia la administración del sitio arqueológico, además de canalizar todos los ingresos a una caja central y mediante planes operativos financiar las mejoras o proyectos, cuyo proceso es indirecto, además se puede realizar convenios para que la administración quede en una asociación o cooperativa, con porcentaje que recibirían por la Administración, se establece boleto de ingreso.

Estas ambigüedades respecto a la Administración ceñido a la propiedad es que debilita la institucionalidad, cumplimiento, desarrollo del sector. Asimismo, otro problema es la debilidad gremial, los grupos de intereses y defensa en el sector Turismo, como son las Cámaras de Turismo, presenta una pobre vida institucional, debido a que los operadores turísticos están direccionados en la recuperación del negocio, cumplir los tributos, priorizar las necesidades familiares, tomar riesgos propios e individuales; ello demuestra la poca cultura de asociatividad, cooperación, barreras de comunicación, imposición de intereses individuales o grupales por encima de los de la comunidad u asociación, lo cual no se busca cambiar en los operadores para lograr un cambio y fortalecer la asociatividad y cooperación, desde Dircetur Apurímac. En el Plan Estratégico Regional de Turismo Apurímac 2018-2025, no se toma en consideración el fortalecimiento gremial, no existe una estrategia, acción estratégica, indicadores para buscar tratar el tema de la agremiación.

\section{DISCUSIÓN}

Tomados los antecedentes, podemos confirmar que las estrategias, pasos claves, para recuperar el sector turismo, dependerá solo y exclusivamente de la iniciativa privada; porque la planta de soporte turística en la región Apurímac, solo tiene tres destinos turísticos desarro- 
llados, los cuales presentan data y presencia en instituciones internacionales, están ubicados en la ciudad de Abancay; los operadores turísticos, tendrán que diversificar su negocio, reaprender, adaptarlo, de tal forma que el levantamiento gradual del aislamiento y confinamiento social, permita la recuperación y reprogramación de viajes, que además asegura la pernoctación y recorrido de circuito. La demanda gradual de visitantes nacionales y extranjeros, hacia la ciudad de Abancay estará condicionada a los servicios de seguridad, sanidad, protección, adicionados a los antiguos paquetes turísticos, complementado al acondicionamiento de la infraestructura, impulso de la promoción en redes sociales y logística de la prestación del servicio que brinden los operadores turísticos, sumado el papel del gremio de operadores turísticos, que es complementario, porque permitirá coordinar políticas, acciones de intervención, defensa de los operadores turísticos en la ciudad de Abancay.

Para las ciudades de Andahuaylas, Aymaraes, Grau, entre otras, de la región Apurímac, los operadores turísticos tendrán que reaprender, adaptar y reorientar hacia otros mercados de visitantes los servicios que prestan, por el motivo que los recursos turísticos, presentan cuellos de botella que impide apalancarse con el operador local, limita su aprovechamiento, además al no tener circuitos definidos, les impide ofertar paquetes turísticos que aseguren una mayor pernoctación, frenando la inyección de gasto del turista hacia la economía local. Para estas ciudades el levantamiento gradual del aislamiento y confinamiento social, solo permitirá iniciar el afianzamiento del turismo local, pero dada las obstrucciones, condiciones de la planta de soporte, la demanda gradual de visitantes nacionales y extranjeros será mínima, además que la consolidación gremial de representación, se mantendrá débil por las incapacidades en operadores turísticos respecto a la asociatividad, cooperación, integración.

Debemos tener presente que, la gestión de la Gerencia Regional de Comercio Exterior y Turismo, durante los últimos seis años, logró aprobar dos proyectos de inversión, con código de inversión pública y presupuesto certificado por más de 20 millones de soles para la ciudad de Abancay, las demás ciudades ubicadas en otras provincias a la fecha no cuentan con proyectos de inversión que favorezcan fortalecer un circuito turístico, la información está contenida en el Plan Estratégico Regional de Turismo Apurímac 2018-2025.

\section{CONCLUSIÓN}

Terminado el análisis de antecedentes, marco normativo, experiencia, entrevistas, discusión, llegamos a tener la siguiente conclusión:

El cuello de botella primordial es el saneamiento físico-legal de los recursos turísticos, es el procedimiento principal, de lo contrario no se podrá obtener dominio, sin ello no se puede inyectar inversión en el entorno local para recuperar, mejorar las instalaciones del recurso turístico. Los cuellos de botella, complementarios son:

1. La accesibilidad y vulneración del recurso turístico;

2. Las limitaciones de jerarquización de los operadores turísticos por no alcanzar requisitos mínimos;

3. La superposición de algunas competencias y funciones de instituciones pertinentes, que hacen duplicar acciones, sumado a la imposición de procesos que frenan el desarrollo del recurso turístico; 
4. El direccionamiento e inclinación de crecimiento, expansión de circuitos turísticos en una ciudad de la región Apurímac.

5. La poca sensibilidad, cooperación para fortalecer el gremio de operadores turísticos.

\section{REFERENCIAS}

Agricultura, M. D. (17 de Abril de 1987). Ley 24656: Ley General de comunidades campesinas. El peruano, pág. 18.

Agricultura, M. d. (17 de Abril de 1987). Ley de Deslinde y Titulación de Territorios Comunales. El Peruano, pág. 19.

Apurímac, G. R. (2018). Plan estratégico regional de Turismo Apurímac 2018-2025. Abancay: GORE Apurímac.

Biosfhere-OMT. (2020). Propuesta de acción para la recuperación empresarial del sector Turístico. Biosfhere.

Carrasco, D. (2005). Metodología de la investigación científica: pautas metodológicas para diseñar y elaborar el proyecto de investigación (primera edición). Editorial San Mar$\cos$

CEPAL. (2020). América latina y el caribe ante la Pandemia del Covid 19: Efectos económicos y sociales. CEPAL.

Cultura, V. M. (2018). Reglamento de intervención arqueológica. El Peruano, pág. 12.

Fidias, G. A. (2012). El Proyecto de Investigacion. Editorial Episteme,C.A.

Hernández, S. R, Fernández, C. C. \& Baptista, L.P. (2014). Metodología de la investigación (sexta edición) Mc Graw Hill.

MINCETUR. (2018). Guía para la actualización e inventario de recursos turísticos. MINCETUR.

Ortiz F. G \& García M. (2008). Metodología de la investigación. LIMUSA.

OMT. (2020). Programa de asistencia técnica para la recuperación del turismo de la crisis del covid 19. (C) Chanwit Whanset - Dreamstime.com.

Promperú. (2020). Ante la crisis, 6 consejos para empresas del sector turismo. Turismo in-Investiga e innova.

PromPerú. (16 de Abril de 2020). Medidas tributarias y financieras. ¿Cómo aprovechar las medidas de la emergencia nacional en el sector Turismo? Prom Perú.

Turismo-Artesanía, D. G. (30 de Marzo de 2020). Reporte mensual Turismo. Boletín de estadísticas de la Dirección General de Investigación y estudios sobre Turismo y Artesanía, pág. 5. https://mincetur.gob.pe

Valle Díaz, F. R. (2020). Potencial de los recursos turísticos como oportunidad de recuperación económica en la región Apurímac, Perú frente al Covid 19. Ciencia Latina-revista Multidisciplinar, 298-323. https://doi.org/10.37811/cl_rcm.v4i2.79. 